\title{
ON RIEMANNIAN MANIFOLDS ADMITTING A FUNCTION WHOSE GRADIENT IS OF CONSTANT NORM II
}

\author{
Dedicated to Professor S. Tanno on his sixtieth birthday
}

TAKASHI SAKAI

\begin{abstract}
We continue to study the metrical structure of complete Riemannian manifolds which admit a smooth function $f$ with $\|\nabla f\| \equiv 1$ for the gradient vector $\nabla f$. We again show that Ricci curvatures controll such metric structure considerably appealing to recent Cheeger-Colding's methods.
\end{abstract}

\section{Introduction}

Let $(M, g)$ be a complete connected smooth Riemannian manifold of dimension $m$ and $f: M \rightarrow \boldsymbol{R}$ a smooth function satisfying

$$
\|\nabla f\| \equiv 1
$$

where $\nabla f$ denotes the gradient vector field of $f$. We denote by $\varphi_{s}, s \in \boldsymbol{R}$ the flow generated by $\nabla f$, and set $Z=f^{-1}(0)$. Then for any $p \in Z$, the curve $c: s \mapsto \varphi_{s}(p)$ is a geodesic such that $d_{M}\left(Z, f^{-1}(s)\right)=L\left(\left.c\right|_{[0, s]}\right)=s=f(c(s))$. Namely, the map $\Phi: X=R \times Z \rightarrow M$ defined by $\Phi(s, z):=\varphi_{s} z$ is a diffeomorphism. Now suppose the Ricci curvature $\operatorname{Ric}_{M}$ of $M$ satisfies

$$
\operatorname{Ric}_{M}(\nabla f, \nabla f) \geq-(m-1) \delta
$$

where $\delta$ is a nonnegative constant. In the previous paper ([S-1]) we showed

THEOREM. For the Laplacian $\Delta f=-g^{i j} \nabla_{i} \nabla_{J} f$ of $f$ we have

$$
|\Delta f| \leq(m-1) \delta \text {. }
$$

Moreover, if $\delta=0$, i.e., $\operatorname{Ric}_{M}(\nabla f, \nabla f) \geq 0$, then $f$ is an affine function and $\Phi: X \rightarrow M$ is an isometry, where $Z:=f^{-1}(0)$ is endowed with the totally geodesic induced Riemannian metric, and $X=R \times Z$ means the Riemannian product.

Next suppose $\delta=1$ and $|\Delta f| \equiv m-1$. Then $\Phi: X \rightarrow M$ is an isometry, where $X$ is endowed with a warped product metric $R \times{ }_{\psi} Z$ with $\psi(t)=\exp ( \pm t)$.

* The author is partially supported by Grant-1n-Aid for Scientific Research (No. 08640118, No. 09640109), Ministry of Education, Science, Sports and Culture (Japan).

Received July 23, 1997; revised March 9, 1998. 
Moreover, $f$ is a Busemann function defined by asymptotic rays $t \mapsto(t, p)$ (or, $t \mapsto(-t, p))$.

In fact, in [S-1] we have assumed that $\operatorname{Ric}_{M} \geq-(m-1) \delta$ instead of (1.2). However, the proof presented there works under the assumption (1.2). See also [S-2] for the case where we take a general warped product as a model.

Now in the present note first we ask what happens for the case where $\operatorname{Ric}_{M}(\nabla f, \nabla f)$ is almost nonnegative. Recently, in their very important papers ([C-1,2,3], [C-C-1,2]) J. Cheeger and T. H. Colding gave new powerful methods to study the structure of complete Riemannian manifolds whose Ricci curvatures are bounded below. In the following we apply their ideas to our rather restricted situation.

We set $\Psi=\Phi^{-1}$, i.e., $\Psi(p)=(f(p), \pi(p))$ with $\varphi_{f(p)}(\pi(p))=p$, where $\pi(p)=\phi_{-f(p)}(p)$ is the foot of the perpendicular of $p$ on $Z$ along a trajectory of $\nabla f$. Then we get

THEOREM 1.1. Let $M$ be a complete connected Riemannian manifold of dimension $m$ admitting a smooth function $f: M \rightarrow \boldsymbol{R}$ with $\|\nabla f\| \equiv 1$. Suppose the Ricci curvature of $M$ satisfies $\operatorname{Ric}_{M} \geq-(m-1) \kappa$ and $\operatorname{Ric}_{M}(\nabla f, \nabla f) \geq-(m-1) \delta$, where $\delta, \kappa$ are positive constants. Then for any $\chi>0, \varepsilon>0$ and $R>0$, there exists $\tau=\tau(m, \chi, \varepsilon, \kappa, R)>0$ such that if $0<\delta<\tau$ then the following holds:

Let $B_{R}(p ; M)$ denote the distance ball in $M$ centered at $p \in Z$. There exists a distance $d_{\chi}$ on $Z$ defined by (2.30) which is almost totally geodesic when restricted to $Z \cap B_{R}(p ; M)$ in the sense that

$$
\left\{\begin{array}{l}
d_{M}\left(z, z^{\prime}\right) \leq d_{\chi}\left(z, z^{\prime}\right) \leq d_{M}\left(z, z^{\prime}\right)+\varepsilon, \\
\gamma_{z z^{\prime}} \subset T_{\varepsilon}(Z ; M) \text { for any } z, z^{\prime} \in Z \cap B_{R}(p ; M),
\end{array}\right.
$$

where $\gamma_{z z^{\prime}}$ is a minimal geodesic in $M$ joining $z$ to $z^{\prime}$ and $T_{\varepsilon}(Z ; M)$ is the $\varepsilon$-tubular neighborhood of $Z$. Now let $d_{X, \chi}$ be the Riemannian product metric on $X:=$ $\boldsymbol{R} \times\left(Z ; d_{\chi}\right)$. Then $\Psi:=\left.\Psi\right|_{B_{R}(p ; M)}: B_{R}(p ; M) \rightarrow X$ satisfies

$$
\left|d_{M}\left(x, x^{\prime}\right)-d_{X, \chi}\left(\Psi(x), \Psi\left(x^{\prime}\right)\right)\right|<\varepsilon,
$$

and for any $y \in B_{R}\left((0, p) ; d_{X, \chi}\right) \subset X$ there exists an $x \in B_{R}(p ; M)$ such that

$$
d_{X, \chi}(y, \Psi(x))<\varepsilon .
$$

In particular, for the Gromov-Hausdorff distance we get

$$
d_{G H}\left(B_{R}(p ; M), B_{R}\left(p ; d_{X, \chi}\right)\right)<\varepsilon .
$$

In fact, Theorem 1.1 holds under the assumption that $\operatorname{Ric}_{M} \geq-(m-1) \kappa$, $\operatorname{Ric}_{M}(\nabla f, \nabla f) \geq-(m-1) \delta$ on $B_{\tilde{R}}(p ; M)$, and that

$$
\frac{1}{\operatorname{vol} B_{\tilde{R}}(p ; M)} \int_{B_{\tilde{R}}(p ; M)}|\Delta f|^{2} d v_{g} \leq(m-1)^{2} \delta^{2}
$$


with e.g., $\tilde{R}=16 R$ for given $R>0$. If we assume that $\operatorname{Ric}_{M} \geq-(m-1) \delta,(1.7)$ follows from a far more general result Theorem 6.62 of [C-C-1]. However, I would like to emphasize that in our restricted situation it is possible to apply geometric part of Cheeger-Colding's method directly to our function $f$ and the proof presented here, which also motivates the following Theorem 1.2, is considerably simpler. In fact, we do not need analytic argument of Cheeger-Colding aproximating distance functions via harmonic functions. In the following we follow the notations of [C-C-1] in principle. However, note that the sign of the Laplacian $\Delta f$ is different from that of Cheeger-Colding's papers.

Next we are concerned with the case where the Ricci curvature of $M$ satisfies

$$
\operatorname{Ric}_{M} \geq-(m-1) \kappa, \quad \operatorname{Ric}_{M}(\nabla f, \nabla f) \geq-(m-1)-\delta
$$

on $B_{\tilde{R}}(p ; M)$, and that $|\Delta f|$ is almost equal to $m-1$ in the sense that

$$
\frac{1}{\operatorname{vol} B_{\tilde{R}}(p ; M)} \int_{B_{\tilde{R}}(p ; M)}|\Delta f \pm(m-1)|^{2} d v_{g}<\delta^{2} .
$$

Then we get

THEOREM 1.2. Let $M$ be a connected complete Riemannian manifold of dimension $m$ admitting a smooth function $f$ with $\|\nabla f\|=1$. Then for any $(1>) \chi>0, \varepsilon>0$ and $R>0$, there exists $\tau=\tau(m, \chi, \varepsilon, \kappa, R)>0$ such that if (1.8) and (1.9) hold for $0<\delta<\tau$ with e.g. $\tilde{R}=30 R$, then we have the following:

There exists a distance $d_{\chi}$ on $Z$ defined by (2.30) which is close to the distance on $Z$ obtained from the induced metric. Let $d_{X, \chi}$ be the distance on $X$ which is the warped product distance $\boldsymbol{R} \times_{\psi}\left(Z, d_{\chi}\right)$ defined by (3.23) with $\psi(t)=\exp ( \pm t)$. Then $\Psi:=\left.\Psi\right|_{B_{R}(p ; M)}, p \in Z=f^{-1}(0)$ satisfies

$$
\left|d_{M}\left(x, x^{\prime}\right)-d_{X, \chi}\left(\Psi(x), \Psi\left(x^{\prime}\right)\right)\right|<\psi(\varepsilon|\chi| R)
$$

and for any $y \in B_{R}\left(p ;\left(X, d_{X, \chi}\right)\right)$ there exists an $x \in B_{R}(p ; M)$ such that

$$
d_{X, \chi}(y, \Psi(x))<\psi(\varepsilon|\chi| R) .
$$

In particular, for the Gromov-Hausdorff distance we get

$$
d_{G H}\left(B_{R}(p ; M), B_{R}\left(p ;\left(X, d_{X \cdot \chi}\right)\right)\right)<\psi(\varepsilon|\chi| R),
$$

where $\psi(\varepsilon|\chi| R)$ means that for fixed $R>0$ we have $\psi(\varepsilon|\chi| R) \downarrow 0$ as $\chi \downarrow 0$ and taking $\varepsilon=\varepsilon(\chi) \downarrow 0$.

In $\S 2$ we give a proof of Theorem 1.1 , and in $\S 3$ we give a proof of Theorem 1.2. As mentioned above our proof much owes to Cheeger-Colding's ideas ([C-C-1]), and our results may be regarded as test cases which verify the power of their methods. The author would like to thank Tobias. H. Colding for some helpful conversations and the referee for some valuable suggestions. 


\section{Almost nonnegative Ricci curvature case}

Let $(M, g)$ be a complete connected Riemannian manifold of dimension $m$. Suppose we have a smooth function $f: M \rightarrow R$ with $\|\nabla f\| \equiv 1$, where $\nabla f$ denotes the gradient vector field of $f$. Now let $\varphi_{s}$ be the flow generated by $\nabla f$. Then for any $p \in M, s \mapsto \varphi_{s}(p)$ is defined for all $s \in \boldsymbol{R}$ and is a geodesic realizing the distance between levels of $f$. Hence the map $\Phi: X:=R \times Z \rightarrow M$ with $Z=f^{-1}(0)$ defined by $\Phi(s, z):=\varphi_{s}(z)$ is a diffeomorphism. Note that at any point $\Phi(s, z) \in M$, tangent vectors $D \Phi_{(s, z)}(0, u)=D \varphi_{s} u$ and $D \Phi_{(s, z)}(\partial / \partial s, 0)=$ $\nabla f(\Phi(s, z))$ are orthogonal to each other. We denote by $\pi: M \rightarrow Z$ the map composed of $\Psi:=\Phi^{-1}$ and the standard projection $R \times Z \rightarrow Z$.

Now suppose that the Ricci curvature $\operatorname{Ric}_{M}$ of $M$ satisfies

$$
\operatorname{Ric}_{M}(\nabla f, \nabla f) \geq-(m-1) \delta,
$$

where $\delta$ is a nonnegative constant. Then we showed in the previous paper ([S]) that the Laplacian $\Delta f$ of $f$ satisfies $|\Delta f| \leq(m-1) \delta$ everywhere. If $\delta=0$, then $f$ is an affine function in the sense that $f \circ \gamma$ is an affine function for any geodesic $\gamma$ in $M$, and $\Phi$ is an isometry between $M$ and the Riemannian product $X:=R \times Z \rightarrow M$ where $Z$ is endowed with the totally geodesic induced metric.

Now we ask whether $\Phi$ is close to an isometry in some appropriate sense when $\delta>0$ is small. First to explain our motivation we consider the special case where $\Delta f$ varies only little in the direction of the gradient of $f$ in the sense that

$$
\nabla f(\Delta f) \leq \delta
$$

and see that $\Phi$ is close to an isometry in Lipschitz sense. In fact, recall the Bochner formula:

$$
\frac{1}{2} \Delta\|\nabla u\|^{2}=\langle\nabla u, \nabla \Delta u\rangle-\operatorname{Ric}_{M}(\nabla u, \nabla u)-\left\|D^{2} u\right\|^{2},
$$

where $D^{2} u$ denotes the Hessian of $u$. Applying this especially to $f$, for which $\|\nabla f\| \equiv 1$ and (2.2) hold, we have

$$
\left\|D^{2} f\right\|^{2} \leq m \delta .
$$

Since $D^{2} f$ restricted to any level of $f$ is the second fundamental form of the level, (2.4) geometrically means that levels of $f$ are almost totally geodesic. Then it follows that for $u \in U_{p} M, p \in Z$

$$
\begin{aligned}
\left|\frac{d}{d s}\left\langle D \varphi_{s} u, D \varphi_{s} u\right\rangle\right| & =2\left|\left\langle\nabla_{\nabla f} D \varphi_{s} u, D \varphi_{s} u\right\rangle\right| \\
& =2\left|D^{2} f\left(D \varphi_{s} u, D \varphi_{s} u\right)\right| \leq 2 \sqrt{m \delta}\left\langle D \varphi_{s} u, D \varphi_{s} u\right\rangle .
\end{aligned}
$$

Integrating the above inequality we get

$$
\exp (-\sqrt{m \delta} s) \leq\left\|D \varphi_{s}(u)\right\| \leq \exp (\sqrt{m \delta} s)
$$

and for the operator norm of $D \Phi_{s, z}: T_{(s, z)} X \rightarrow T_{\Phi(s, z)} M$, it follows that 


$$
\exp (-\sqrt{m \delta} s) \leq\left\|D \Phi_{(s, z)}\right\| \leq \exp (\sqrt{m \delta} s) .
$$

Therefore, we have the following result.

Proposition 2.1. Suppose $M$ and $f$ with $\|\nabla f\|=1$ satisfy (2.1) and (2.2). Then for any $\varepsilon>0$ and any $R>0$ there exists $\tau=\tau(\varepsilon, m, R)>0$ such that if $0<\delta<\tau$ in (2.1) and (2.2), then we get for $\Phi$ restricted to $B_{R}((0, p) ; X) \subset X$, $p \in Z$

$$
(1-\varepsilon) d_{X}(x, y) \leq d_{M}(\Phi(x), \Phi(y)) \leq(1+\varepsilon) d_{X}(x, y),
$$

where $Z=f^{-1}(0)$ is endowed with the induced metric and $X=\boldsymbol{R} \times Z$ is the Riemannian product. Furthermore, levels of $f$ are almost totally geodesic in the sense of (2.4).

In fact, we get Proposition 2.1 under the assumption that (2.1), (2.2) hold on a larger concentric ball $B_{2 R}(p ; M)$. Of course the assumption (2.2) is geometrically not nice, and we turn to the original problem, namely, consider $M$ admitting a function $f$ with $\|\nabla f\|=1$, whose Ricci curvature satisfies

$$
\operatorname{Ric}_{M} \geq-(m-1) \kappa, \quad \operatorname{Ric}_{M}(\nabla f, \nabla f) \geq-(m-1) \delta,
$$

where $\kappa, \delta$ are positive constants. We assume that $\delta<1, \kappa>1$ in (2.7). Recall that a map $\Psi: X \rightarrow Y$ between metric spaces $X, Y$ is called an $\varepsilon$-Hausdorff approximation if we have

$$
\left\{\begin{array}{l}
\left|d_{Y}\left(\Psi(x), \Psi\left(x^{\prime}\right)\right)-d_{X}\left(x, x^{\prime}\right)\right|<\varepsilon \quad \text { for } x, x^{\prime} \in X, \\
Y \subset T_{\varepsilon}(\Psi(X)),
\end{array}\right.
$$

where we set $T_{\varepsilon}(\Psi(X)):=\{y \in Y \mid d(y, \Psi(X))<\varepsilon\}$. We say that the GromovHausdorff distance $d_{G H}(X, Y)<\varepsilon$ if there exists an $\varepsilon$-Hausdorff approximation $\Psi: X \rightarrow Y$.

In the following we show that for any $R>0$ and $\varepsilon>0, \Psi:=$ $\Phi^{-1}: M \rightarrow R \times Z$ is roughly an $\varepsilon$-Hausdorff approximation when restricted to a distance ball $B_{R}(p ; M)$, if $\delta>0$ is sufficiently small and $Z$ is endowed with a distance which is close to the induced metric in some sense.

We begin with the following lemma.

LEMMA 2.2. There exists $C=C(m, \kappa, R)>0$ such that for $p \in M$ we have

$$
\frac{1}{\operatorname{vol} B_{R}(p)} \int_{B_{R}(p)}\left\|D^{2} f\right\|^{2} d v_{g} \leq C \delta
$$

Proof. By the Bochner formula (2.3) and the assumption (2.1) we get

$$
\begin{aligned}
\left\|D^{2} f\right\|^{2} & =\langle\nabla f, \nabla \Delta f\rangle-\operatorname{Ric}_{M}(\nabla f, \nabla f) \\
& \leq\langle\nabla f, \nabla \Delta f\rangle+(m-1) \delta .
\end{aligned}
$$


Now take a cut off function $\phi: M \rightarrow[0,1]$ such that

$$
\phi\left|B_{R}(p) \equiv 1, \quad \operatorname{supp} \phi \subset B_{2 R}(p), \quad\|\nabla \phi\|,\right| \Delta \phi \mid \leq c(m, \kappa, R)
$$

(see [C-C-1], Theorem 6.33). Then we have

$$
\int_{B_{2 R}(p)} \phi\left\|D^{2} f\right\|^{2} d v_{g} \leq \int_{B_{2 R}(p)} \phi\langle\nabla f, \nabla \Delta f\rangle d v_{g}+(m-1) \delta \int_{B_{2 R}(p)} \phi d v_{g} .
$$

Since

$$
\phi\langle\nabla f, \nabla \Delta f\rangle=\operatorname{div}(\phi \Delta f \nabla f)-\langle\nabla \phi, \nabla f\rangle \Delta f+\phi(\Delta f)^{2}
$$

holds, it follows from the Green formula that

$$
\begin{aligned}
& \frac{1}{\operatorname{vol} B_{R}(p)} \int_{B_{R}(p)}\left\|D^{2} f\right\|^{2} d v_{g} \leq \frac{1}{\operatorname{vol} B_{R}(p)} \int_{B_{2 R}(p)} \phi\left\|D^{2} f\right\|^{2} d v_{g} \\
& \quad \leq(m-1) \delta\{\sup \|\nabla \phi\|+(m-1) \delta+1\} \frac{\operatorname{vol} B_{2 R}(p)}{\operatorname{vol} B_{R}(p)} \\
& \quad \leq(m-1) \delta C_{1}(m, \kappa, R) \frac{v_{-\kappa}^{m}(2 R)}{v_{-\kappa}^{m}(R)}
\end{aligned}
$$

where $v_{-\kappa}^{m}(r)$ denotes the volume of distance $r$-ball in the simply connected space form of constant curvature $-\kappa$, and we have used the Bishop-Gromov volume comparison theorem. This completes the proof of the lemma.

Modifying the proof above slightly we get the following:

Remark. (2.8) holds under a weaker assumption that

$$
\operatorname{Ric}_{M} \geq-(m-1) \kappa, \quad \operatorname{Ric}_{M}(\nabla f, \nabla f) \geq-(m-1) \delta \quad \text { on } B_{2 R}(p ; M),
$$

and

$$
\frac{1}{\operatorname{vol} B_{2 R}(p ; M)} \int_{B_{2 R}(p ; M)}|\Delta f|^{2} d v_{g} \leq(m-1)^{2} \delta^{2} .
$$

Now to compare $M$ with the Riemannian product space $X=R \times Z$ we recall some fundamental metrical properties of $X$ :

$$
\begin{aligned}
d_{X}\left(\left(r_{1}, z_{1}\right),\left(r_{2}, z_{2}\right)\right) & =\sqrt{\left(r_{2}-r_{1}\right)^{2}+d_{Z}\left(z_{1}, z_{2}\right)^{2}} \\
& =: \rho\left(r_{1}, r_{2}, d_{Z}\left(z_{1}, z_{2}\right)\right) .
\end{aligned}
$$

Then we have the triangle inequality

$$
\rho\left(r_{1}, r_{3}, v_{1}+v_{2}\right) \leq \rho\left(r_{1}, r_{2}, v_{1}\right)+\rho\left(r_{2}, r_{3}, v_{2}\right),
$$

where equality holds choosing $r_{2}$ suitably. Let $\underline{r}: \boldsymbol{R} \times Z \rightarrow \boldsymbol{R}, \underline{\pi}: \boldsymbol{R} \times Z \rightarrow Z$ be canonical projections. Suppose $\underline{x}_{l}, \underline{y}_{l} \in \boldsymbol{R} \times \bar{Z}(i=1,2)$ satisfy $\underline{\pi}\left(\underline{x}_{1}\right)=\underline{\pi}\left(\underline{y}_{1}\right)$, $\underline{\pi}\left(\underline{x}_{2}\right)=\underline{\pi}\left(\underline{y}_{2}\right)$. Then we have 


$$
\begin{aligned}
d_{X}\left(\underline{y}_{1}, \underline{y}_{2}\right) & =\sqrt{\left.d_{X}\left(\underline{x}_{1}, \underline{x}_{2}\right)^{2}+\left(\underline{r}_{2}\right)-\underline{r}\left(\underline{y}_{1}\right)\right)^{2}-\left(\underline{r}\left(\underline{x}_{2}\right)-\underline{r}\left(\underline{x}_{1}\right)\right)^{2}} \\
& \left.\left.\left.=: Q\left(\underline{r}_{1} \underline{x}_{1}\right), \underline{r}_{(} \underline{y}_{1}\right), \underline{r}\left(\underline{x}_{2}\right), \underline{r}_{(} \underline{y}_{2}\right), d_{X}\left(\underline{x}_{1}, \underline{x}_{2}\right)\right)
\end{aligned}
$$

and

$$
\rho\left(r_{1}, r_{2}, v\right)=Q\left(0, r_{1}, 0, r_{2}, v\right) .
$$

Now if $Z$ is a Riemannian manifold and $\underline{\gamma}(s)=(\underline{r}(s), \underline{c}(s)), 0 \leq s \leq l$, is a geodesic of $X=\boldsymbol{R} \times Z$ parametrized by arclength, then $\underline{\theta}(s):=\angle(\underline{\hat{\gamma}}(s), \partial / \partial r)$ is constant, and we have

$$
\left\{\begin{array}{l}
\underline{r}(\underline{\gamma}(s))=r_{0}+\left(r_{l}-r_{0}\right) \frac{s}{l}=: \underline{\mathscr{U}}(s)\left(=\underline{\mathscr{U}}\left(s ; r_{0}, r_{l}, l\right)\right) \\
\cos \underline{\theta}(s)=\underline{\mathscr{U}}^{\prime}(s)=\frac{r_{l}-r_{0}}{l}, \quad \underline{\theta}(s)=: \Theta\left(r_{0}, r_{l}, l\right),
\end{array}\right.
$$

where we set $r_{0}=\underline{r}(0), r_{l}=\underline{r}(l)$. Note that $\underline{r}, \underline{\theta}$ only depend on $l$ and the boundary condition $r_{0}, r_{l}$, and do not depend on Riemannian metrics on $Z$.

Now we turn to our Riemannian manifold $M$. For a minimal geodesic $\gamma_{y_{1} y_{2}}$ joining $y_{1}$ to $y_{2}$ in $M$ parametrized by arclength, we set

$$
\mathscr{U}(s)\left(=\mathscr{U}\left(s ; y_{1}, y_{2}\right)\right):=f\left(\gamma_{y_{1} y_{2}}(s)\right) \text {. }
$$

Then we easily see that

$$
\left\{\begin{array}{l}
\mathscr{U}^{\prime}(s)=\left\langle\nabla f, \dot{\gamma}_{y_{1} y_{2}}(s)\right\rangle=: \cos \theta(s), \quad \theta(s)=\theta\left(s ; y_{1}, y_{2}\right) \\
\mathscr{U}^{\prime \prime}(s)=D^{2} f\left(\dot{\gamma}_{y_{1} y_{2}}(s), \dot{\gamma}_{y_{1} y_{2}}(s)\right) .
\end{array}\right.
$$

Now we want to compare $\mathscr{U}(s)$ with $\underline{\mathscr{U}}(s)\left(:=\underline{\mathscr{U}}\left(s ; r_{0}, r_{l}, l\right)\right)$ setting $r_{0}=f\left(y_{1}\right)=$ $\mathscr{U}(0), r_{l}=f\left(y_{2}\right)=\mathscr{U}(l), l=d\left(y_{1}, y_{2}\right)$. First we assume that

$$
\int_{0}^{l}\left|\mathscr{U}^{\prime \prime}(s)\right| d s<\varepsilon_{2}
$$

Recall that

$$
\begin{aligned}
& \underline{\mathscr{U}}^{\prime \prime}(s)=0, \quad \underline{\mathscr{U}}^{\prime}(s)=\frac{r_{l}-r_{0}}{l} \\
& \mathscr{U}(0)=\underline{\mathscr{U}}(0), \quad \mathscr{U}(l)=\underline{\mathscr{U}}(l) .
\end{aligned}
$$

Then from

$$
\left|\mathscr{U}^{\prime}(s)-\mathscr{U}^{\prime}(0)\right| \leq \int_{0}^{s}\left|\mathscr{U}^{\prime \prime}(s)\right| d s<\varepsilon_{2} \quad(0 \leq s \leq l)
$$

we get

$$
\mathscr{U}^{\prime}(0)-\varepsilon_{2} \leq \frac{r_{l}-r_{0}}{l}=\frac{1}{l} \int_{0}^{l} \mathscr{U}^{\prime}(s) d s \leq \mathscr{U}^{\prime}(0)+\varepsilon_{2},
$$


namely,

$$
\left|\mathscr{U}^{\prime}(0)-\frac{r_{l}-r_{0}}{l}\right| \leq \varepsilon_{2} .
$$

Therefore, it follows that

$$
\left|\mathscr{U}^{\prime}(s)-\underline{\mathscr{U}}^{\prime}(s)\right| \leq 2 \varepsilon_{2}, \quad|\mathscr{U}(s)-\underline{\mathscr{U}}(s)| \leq 2 \varepsilon_{2} s .
$$

Now to compare $\mathscr{U}(s)$ and $\underline{U}(s)$ for general $\gamma_{y_{1} y_{2}}$ we recall the following result which follows directly from [C-C-1], Theorem 2.11, and converts (2.8) into the corresponding statement on a family of minimal geodesics. Such an idea was first introduced by T. H. Colding to get an $L_{2}$-Toponogov comparison theorem for manifolds with Ricci curvatures bounded below, and has many important applications ([C-1,2,3]).

LEMMA 2.3. Suppose that

$$
\frac{1}{\operatorname{vol} B_{4 R_{1}}(p)} \int_{B_{4 R_{1}}(p)}\left\|D^{2} f\right\| d v_{g} \leq \varepsilon_{1} .
$$

Then there exists $C\left(m, \kappa, R_{1}\right)>0$ such that the following holds:

$$
\begin{aligned}
& \frac{1}{\left(\operatorname{vol} B_{2 R_{1}}(p)\right)^{2}} \int_{B_{2 R_{1}}(p) \times B_{2 R_{1}}(p)} d v_{g \oplus g} \int_{0}^{l}\left\|D^{2} f\right\| \circ \gamma_{y_{1} y_{2}}(s) d s \\
& \quad \leq C\left(m, \kappa, R_{1}\right) \varepsilon_{1},
\end{aligned}
$$

where we set $l=d\left(y_{1}, y_{2}\right)$. Note that $D^{2} f\left(\dot{\gamma}_{y_{1} y_{2}}(s), \dot{\gamma}_{y_{1} y_{2}}(s)\right)=\mathscr{U}^{\prime \prime}\left(s ; y_{1}, y_{2}\right)$.

To be precise, in (2.18) we should integrate over the set $B \subset B_{2 R_{1}}(p) \times$ $B_{2 R_{1}}(p)$ of full measure such that for any $\left(y_{1}, y_{2}\right) \in B$ there exists a unique minimal geodesic $\gamma_{y_{1} y_{2}}$ parametrized by arclength. Also note that from Lemma 2.2 with Cauchy-Schwarz inequality, the assumption (2.17) holds for any $4 R_{1}>0$ taking $\delta=\delta\left(m, \kappa, R_{1}\right)$ in (2.1) sufficiently small.

This lemma roughly means that for almost all $y_{1} \in B_{2 R_{1}}(p)$ and $y_{2} \in B_{2 R_{1}}(p)$ we have $\int_{0}^{l}\left|\mathscr{U}^{\prime \prime}\left(s ; y_{1}, y_{2}\right)\right| d s<\varepsilon_{2}$, where "almost all" means "except for a set of very small volume". To be more precise, we denote by $D_{\varepsilon_{2}}\left(y_{1}\right), y_{1} \in B_{2 R_{1}}(p)$, the set of points $y_{2} \in B_{2 R_{1}}(p)$ such that there exists a unique minimal geodesic $\gamma_{y_{1} y_{2}}$ joining $y_{1}$ to $y_{2}$ parametrized by arclength, and that $\int_{0}^{l}\left|\mathscr{U}^{\prime \prime}(s)\right| d s<\varepsilon_{2}$ $\left(\mathscr{U}^{\prime \prime}(s):=\mathscr{U}^{\prime \prime}\left(s ; y_{1}, y_{2}\right)\right)$ holds. Note that $\gamma_{y_{1} y_{2}} \subset B_{4 R_{1}}(p)$. Next we set

$$
Q_{\varepsilon_{2}}:=\left\{y_{1} \in B_{2 R_{1}}(p) \mid \operatorname{vol} D_{\varepsilon_{2}}\left(y_{1}\right) \geq\left(1-\varepsilon_{2}\right) \operatorname{vol} B_{2 R_{1}}(p)\right\} \text {. }
$$

Then from the lemma above it easily follows that for any $\varepsilon_{2}>0$ there exists $\tau=\tau\left(\varepsilon_{2}, m, \kappa, R_{1}\right)>0$ such that if $0<\varepsilon_{1}<\tau$ and

$$
\frac{1}{\operatorname{vol} B_{4 R_{1}}(p)} \int_{B_{4 R_{1}}(p)}\left\|D^{2} f\right\| d v_{g}<\varepsilon_{1}
$$


holds, we have

$$
\operatorname{vol} Q_{\varepsilon_{2}} \geq\left(1-\varepsilon_{2}\right) \operatorname{vol} B_{2 R_{1}}(p) \text {. }
$$

Note that for $y_{1} \in Q_{\varepsilon_{2}}, y_{2} \in D_{\varepsilon_{2}}\left(y_{1}\right)$ we have

$$
|\mathscr{U}(s)-\underline{\mathscr{U}}(s)|<2 \varepsilon_{2} s, \quad\left|\mathscr{U}^{\prime}(s)-\underline{\mathscr{U}}^{\prime}(s)\right|<2 \varepsilon_{2}
$$

for $\mathscr{U}(s):=\mathscr{U}\left(s ; y_{1}, y_{2}\right)$ by $(2.15)$, (2.16). Summing up we get the following:

LEMMA 2.4. For any $\varepsilon_{2}>0$ there exists $\tau=\tau\left(\varepsilon_{2}, m, \kappa, R_{1}\right)>0$ such that if

$$
\frac{1}{\operatorname{vol} B_{4 R_{1}}(p)} \int_{B_{4 R_{1}}(p)}\left\|D^{2} f\right\| d v_{g}<\varepsilon_{1}
$$

holds for $0<\varepsilon_{1}<\tau$, then we have for $y_{1} \in Q_{\varepsilon_{2}}, y_{2} \in D_{\varepsilon_{2}}\left(y_{1}\right)$

$$
\left\{\begin{array}{l}
\left|f\left(\gamma_{y_{1} y_{2}}(s)\right)-\underline{\mathscr{U}}\left(s ; f\left(y_{1}\right), f\left(y_{2}\right), l\right)\right|<2 \varepsilon_{2} l, \\
\left|\cos \theta\left(s ; y_{1}, y_{2}\right)-\cos \Theta\left(f\left(y_{1}\right), f\left(y_{2}\right), l\right)\right|<2 \varepsilon_{2}, \\
\int_{0}^{l}\left|\cos \theta\left(s ; y_{1}, y_{2}\right)-\cos \Theta\left(f\left(y_{1}\right), f\left(y_{2}\right), l\right)\right| d s<8 \varepsilon_{2} R_{1},
\end{array}\right.
$$

where we again set $l=d\left(y_{1}, y_{2}\right)$.

Note that for any fixed $y \in Q_{\varepsilon_{2}}$, from (2.15) and (2.16) we get for almost all $z \in B_{2 R_{1}}(p)$

$$
\left|\mathcal{U}^{\prime}(d(y, z) ; y, z)-\underline{\mathscr{U}}^{\prime}(d(y, z) ; f(y), f(z), d(y, z))\right|<2 \varepsilon_{2}
$$

namely,

$$
\begin{aligned}
\frac{1}{\operatorname{vol} B_{2 R_{1}}(p)} & \int_{z \in B_{2 R_{1}}(p)} l\left|\mathscr{U}^{\prime}(l ; y, z)-{\underline{U^{\prime}}}^{\prime}(l ; f(y), f(z), l)\right| d v_{g} \\
& <16 \varepsilon_{2} R_{1},
\end{aligned}
$$

where we set $l=d(y, z)$. Here also note that $\left|\mathscr{U}^{\prime}(s)\right|,\left|\underline{\mathcal{U}^{\prime}}(s)\right| \leq 1$.

Now we give our version of [C-C-1], Proposition 2.80 .

LEMMA 2.5. For any $\varepsilon>0$ there exists $\zeta=\zeta\left(\varepsilon, m, \kappa, R_{1}\right)>0$ such that if

$$
\frac{1}{\operatorname{vol} B_{4 R_{1}}(p)} \int_{B_{4 R_{1}}(p)}\left\|D^{2} f\right\| d v_{g}<\varepsilon_{1}
$$

holds for $0<\varepsilon_{1}<\zeta$, then we have

$$
\left|d_{M}\left(y_{1}, y_{2}\right)-Q\left(f\left(x_{1}\right), f\left(y_{1}\right), f\left(x_{2}\right), f\left(y_{2}\right), d_{M}\left(x_{1}, x_{2}\right)\right)\right|<\varepsilon
$$

for any $x_{1}, x_{2}, y_{1}, y_{2} \in B_{R_{1}}(p)$ with $f\left(y_{i}\right)-f\left(x_{\imath}\right)=d\left(x_{\imath}, y_{i}\right)(i=1,2)$. 
Since we follow the proof of Proposition 2.80 of [C-C-1] in principle, we give a sketch. Considering a minimal geodesic $\gamma_{x_{1} y_{2}}$, which is contained in $B_{2 R_{1}}(p ; M)$, it suffices to show our claim in the case of $x_{1}=y_{1}=x$. Set $\lambda=\gamma_{x_{2} y_{2}}$ and first assume that $x \in Q_{\varepsilon_{2}}, \quad \lambda \subset D_{\varepsilon_{2}}(x)$. Then setting $l(s):=d(x, \lambda(s))$, $d:=d\left(x_{2}, y_{2}\right)$ we have

$$
\cos \theta(l(s) ; x, \lambda(s))=\mathscr{U}^{\prime}(l(s) ; x, \lambda(s))
$$

and

$$
\int_{0}^{l(s)}\left|\mathscr{U}^{\prime \prime}(t ; x, \lambda(s))\right| d t<\varepsilon_{2} .
$$

It follows from (2.20) and $\cos \Theta(f(x), f(\lambda(s)), l(s))=(f(\lambda(s))-f(x)) / l(s)=$ $(s+a) / l(s)$ with $a:=f\left(x_{2}\right)-f(x)$ that for any $\varepsilon_{2}>0$

$$
\left|\cos \theta(l(s) ; x, \lambda(s))-\frac{s+a}{l(s)}\right|<2 \varepsilon_{2},
$$

if we take $\varepsilon_{1}>0$ sufficiently small.

On the other hand, in the model space $R \times Z$ take $\underline{x}, \underline{x}_{2}, \underline{y}_{2}$ such that

$$
\begin{aligned}
& \underline{r}(\underline{x})=f(x), \quad \underline{r}\left(\underline{x}_{2}\right)=f\left(x_{2}\right), \quad \underline{r}\left(\underline{y}_{2}\right)=f\left(y_{2}\right) \\
& d\left(\underline{x}_{2}, \underline{y}_{2}\right)=d=\underline{r}\left(\underline{y}_{2}\right)-\underline{r}\left(\underline{x}_{2}\right), \quad d\left(\underline{x}, \underline{x}_{2}\right)=l(0) .
\end{aligned}
$$

Let $\underline{\lambda}$ be a radial geodesic joining $\underline{x}_{2}$ to $\underline{y}_{2}$, and set $\underline{l}(s):=d(\underline{x}, \underline{\lambda}(s))$. Note that $\underline{l}(0)=l(0)=d_{M}\left(x, x_{2}\right)$ and we have

$$
\underline{l}(s)=\sqrt{(s+a)^{2}+b^{2}}, \quad \underline{l}^{\prime}(s)=\frac{s+a}{\underline{l}(s)}
$$

with $a^{2}+b^{2}=l(0)^{2}$. Then note that

$$
l(d)=d_{M}\left(x, y_{2}\right) \quad \text { and } \quad \underline{l}(d)=Q\left(f(x), f(x), f\left(x_{2}\right), f\left(y_{2}\right), d_{M}\left(x, x_{2}\right)\right) .
$$

Now from the first variation formula we have

$$
l^{\prime}(s)=\cos \theta(l(s) ; x, \lambda(s)), \quad \underline{l}^{\prime}(s)=\cos \Theta(f(x), f(\lambda(s)), \underline{l}(s)) .
$$

Then it follows from (2.23) that $\left|l(s) l^{\prime}(s)-\underline{l}^{\prime}(s) \underline{l}(s)\right|=\left|l(s) l^{\prime}(s)-(s+a)\right| \leq$ $2 \varepsilon_{2}(s+l(0))$. Integrating this inequality we get

$$
\left|l(d)^{2}-\underline{l}(d)^{2}\right|<\varepsilon_{2}\left(l(0) d+\frac{d^{2}}{2}\right) .
$$

Therefore,

$$
|l(d)-\underline{l}(d)|<\psi\left(\varepsilon_{2} \mid R_{1}\right),
$$

where $\psi\left(\varepsilon_{2} \mid R_{1}\right)$ means that for fixed $R_{1}>0, \psi\left(\varepsilon_{2} \mid R_{1}\right) \downarrow 0$ as $\varepsilon_{2} \downarrow 0$.

Now in general case we need some approximation argument. Let $x=x_{1}=y_{1}, x_{2}, y_{2} \in B_{R_{1}}(p)$. We may assume that $d\left(x_{2}, y_{2}\right)>\eta$ for a fixed 
sufficiently small $\eta>0$. Taking $\varepsilon_{2}>0$ sufficiently small we may choose $y \in B_{\eta^{3}}(x) \cap Q_{\eta^{3}}, q \in B_{\eta^{3}}\left(x_{2}\right), w \in B_{\eta^{3}}\left(y_{2}\right) \cap D_{\eta^{3}}(q)$ by virtue of (2.19) and the Bishop-Gromov volume comparison theorem. Set

$$
\lambda=\gamma_{q w}, \quad l(s)=d(y, \lambda(s)), \quad d=d(q, w) .
$$

Now applying Theorem 2.11 of [C-C-1] to (2.21) as in Lemma 2.3, we may assume in the above that

$$
\int_{0}^{d} l(s)\left|\mathscr{U}^{\prime}(l(s) ; y, \lambda(s))-\underline{\mathscr{U}}^{\prime}(l(s) ; f(y), f(\lambda(s)), l(s))\right| d s<\eta^{2},
$$

where $\underline{\mathcal{U}}^{\prime}(l(s) ; f(y), f(\lambda(s)), l(s))=\cos \Theta(f(y), f(\lambda(s)), l(s))=(f(\lambda(s))-f(y)) / l(s)$ in this case. On the other hand, since $w \in D_{\eta^{3}}(q)$, noting that $d>\eta-2 \eta^{3}$ and (2.16) we have

$$
\left\{\begin{array}{l}
\left|f(\lambda(s))-\left(f\left(x_{2}\right)+s\right)\right|<2 \varepsilon_{2} s+5 \eta^{3} \\
|1-\langle\dot{\lambda}(s), \nabla f(\lambda(s))\rangle|<2 \varepsilon_{2}+5 \eta^{2}
\end{array}\right.
$$

Then we set $\alpha(s):=\angle\left(\dot{\gamma}_{y \lambda(s)}(l(s)), \dot{\lambda}(s)\right)$ and note that $l^{\prime}(s)=\cos \alpha(s)$ and

$$
\left|\cos \alpha(s)-\mathscr{U}^{\prime}(l(s) ; y, \lambda(s))\right| \leq\|\dot{\lambda}(s)-\nabla f(\lambda(s))\|<2 \sqrt{\varepsilon_{2}+2 \eta^{2}} .
$$

It follows from (2.25) that

$$
\begin{aligned}
& \int_{0}^{d} l(s)|\cos \alpha(s)-\cos \Theta(f(y), f(\lambda(s)), l(s))| d s \\
& <\psi\left(\varepsilon_{2}, \eta \mid R_{1}\right),
\end{aligned}
$$

where $\psi\left(\varepsilon_{2}, \eta \mid R_{1}\right) \downarrow 0$ as $\varepsilon_{2}, \eta \downarrow 0$ for fixed $R_{1}>0$. Considering the same situation in the model space $X$ with $\underline{l}(0)=l(0)$ etc. as before, we have

$$
\begin{aligned}
& \left.\int_{0}^{d} \underline{l}(s) \mid \cos \underline{\alpha}(s)-\cos \Theta(\underline{f} \underline{y}), \underline{f}(\underline{\lambda}(s)), \underline{l}(s)\right) \mid d s \\
& <\psi\left(\varepsilon_{2}, \eta \mid R_{1}\right),
\end{aligned}
$$

where we also denote $\underline{f}(\underline{y})=\underline{r}(\underline{y})$ etc. Here note that

$$
\begin{gathered}
|l(s) \cos \Theta(f(y), f(\lambda(s)), l(s))-\underline{l}(s) \cos \Theta(\underline{f}(\underline{y}), \underline{f}(\underline{\lambda}(s)), \underline{l}(s))| \\
=|f(\lambda(s))-\underline{f}(\underline{\lambda}(s))|<\psi\left(\varepsilon_{2}, \eta \mid R_{1}\right) .
\end{gathered}
$$

Now by the first variation formula we get

$$
\frac{1}{2}\left(l(s)^{2}-\underline{l}(s)^{2}\right)^{\prime}=l(s) \cos \alpha(s)-\underline{l}(s) \cos \underline{\alpha}(s)
$$

almost everywhere for $0 \leq s \leq l$. Then integrating the equation above as before we complete the proof of Lemma 2.5 . 
We note that (2.22) holds under the condition that $\left|f\left(y_{i}\right)-f\left(x_{i}\right)\right|=d\left(x_{l}, y_{i}\right)$.

Now we turn to the proof of Theorem 1.1. Recall that we have a diffeomorphism $\Psi: M \rightarrow X=R \times Z$ with $Z=f^{-1}(0)$ defined by $\Psi(x)=(f(x), \pi(x))$ with $x=\varphi_{f(x)}(\pi(x))$. Note that $Z$ is connected. We restrict $\Psi$ to a distance ball $B_{R}(p ; M), p \in Z$. First we consider the induced distance $\tilde{d}_{Z}$ on $Z$ from $d_{M}$, i.e., $\tilde{d}_{Z}\left(z, z^{\prime}\right)=d_{M}\left(z, z^{\prime}\right)$, and let $X$ be endowed with the product metric $d_{X}$ (see (2.11)). Then noting that $d_{X}\left(\Psi(x), \Psi\left(x^{\prime}\right)\right)=Q\left(0, f(x), 0, f\left(x^{\prime}\right), \tilde{d}_{Z}\left(\pi(x), \pi\left(x^{\prime}\right)\right)\right)$ and $\pi(x), \pi\left(x^{\prime}\right) \in B_{2 R}(p)$, for any $\varepsilon>0$ we get from Lemma 2.5 with $R_{1}=2 R$

$$
\left|d_{M}\left(x, x^{\prime}\right)-d_{X}\left(\Psi(x), \Psi\left(x^{\prime}\right)\right)\right|<\varepsilon
$$

if we take $\varepsilon_{1}$ sufficiently small. Next we see that the above induced distance $\tilde{d}_{Z}$ on $Z$ is close to the distance defined from the induced Riemannian metric on $Z$ and is almost totally geodesic.

Indeed, for $z, z^{\prime} \in Z \cap B_{R}(p ; M)$ take a minimal geodesic $\gamma=\gamma_{z z^{\prime}}:[0, l] \rightarrow M$ in $M$ with $l=d_{M}\left(z, z^{\prime}\right)$. Then in view of (2.16), $|f(\gamma(s))|$ should be small. In fact, for any $0 \leq s \leq l$ we set $w=\pi(\gamma(s))$ and get

$$
d_{M}(z, w)+d_{M}\left(w, z^{\prime}\right) \geq d_{M}\left(z, z^{\prime}\right)=d_{M}(z, \gamma(s))+d_{M}\left(\gamma(s), z^{\prime}\right) .
$$

From (2.12), (2.22) it follows that

$$
\begin{gathered}
d_{M}(z, \gamma(s)) \geq \sqrt{d_{M}(z, w)^{2}+f(\gamma(s))^{2}}-\varepsilon, \\
d_{M}\left(z^{\prime}, \gamma(s)\right) \geq \sqrt{d_{M}\left(z^{\prime}, w\right)^{2}+f(\gamma(s))^{2}}-\varepsilon .
\end{gathered}
$$

Then adding these two inequalities we easily obtain

$$
|f(\gamma(s))|=d_{M}(\gamma(s), Z)<2 \sqrt{\varepsilon(\varepsilon+R)}=: \varepsilon_{3},
$$

namely, $\gamma \subset T_{\varepsilon_{3}}(Z)$. Next take a subdivision $\left\{\gamma\left(s_{i}\right) ; s_{i}=i l / N, i=0, \ldots, N\right\}$ of $\gamma$, and note that $\pi\left(\gamma\left(s_{i}\right)\right) \in B_{3 R}(p)$. Then from Lemma 2.5 with $R_{1}=4 R$ we obtain

$$
\tilde{d}_{Z}\left(\pi\left(\gamma\left(s_{i}\right)\right), \pi\left(\gamma\left(s_{i+1}\right)\right)\right)<\varepsilon+d_{M}\left(\gamma\left(s_{i}\right), \gamma\left(s_{i+1}\right)\right)=\varepsilon+\frac{l}{N} .
$$

It follows that

$$
\sum_{l=0}^{N-1} \tilde{d}_{Z}\left(\pi\left(\gamma\left(s_{i}\right)\right), \pi\left(\gamma\left(s_{i+1}\right)\right)\right)<\tilde{d}_{Z}\left(z, z^{\prime}\right)+N \varepsilon
$$

Now for $\chi>0$ we define a metric $d_{\chi}$ on $Z$ by

$$
\begin{gathered}
d_{\chi}\left(z, z^{\prime}\right):=\inf \left\{\sum_{l=0}^{N_{1}-1} \tilde{d}_{Z}\left(z_{l}, z_{l+1}\right) \mid z_{\imath} \in Z\left(i=0, \ldots, N_{1}-1\right),\right. \\
\left.z_{0}=z, z_{N_{1}}=z^{\prime}, \tilde{d}_{Z}\left(z_{l}, z_{l+1}\right)<\chi\right\} .
\end{gathered}
$$


Then for any $\chi>0$, choose $\varepsilon>0$ and a positive integer $N:=N(\chi, R)$ so that $\varepsilon+(2 R / N)<\chi$. It follows that $\tilde{d}_{Z}\left(\pi\left(\gamma\left(s_{i}\right), \pi\left(\gamma\left(s_{i+1}\right)\right)\right)<\chi\right.$, and

$$
\left\{\begin{array}{l}
\tilde{d}_{Z}\left(z, z^{\prime}\right) \leq d_{\chi}\left(z, z^{\prime}\right) \leq \tilde{d}_{Z}\left(z, z^{\prime}\right)+\varepsilon_{4} \\
\gamma_{z z^{\prime}} \subset T_{\varepsilon_{4}}(Z ; M)
\end{array}\right.
$$

with $\varepsilon_{4}:=\max \left\{\varepsilon_{3}, N \varepsilon\right\}$. Because of $(2.31)$ we call $d_{\chi}$ an almost $\varepsilon_{4}$-totally geodesic metric. Then we endow $X$ with the product metric $d_{X, \chi}=d_{R} \times d_{\chi}$, and from (2.28) we get

$$
\left|d_{M}\left(x, x^{\prime}\right)-d_{X, \chi}\left(\Psi(x), \Psi\left(x^{\prime}\right)\right)\right|<\varepsilon+\varepsilon_{4}:=\varepsilon_{5},
$$

for $x, x^{\prime} \in B_{R}(p ; M)$. For the above distance $d_{X, \chi}$ on $X$, if $R \leq d_{X, \chi}(p, y)<$ $R+\varepsilon_{5}$, then considering a minimal geodesic in $M$ joining $p$ to $\Phi(y)$ and noting (2.32) we see that there exists $y^{\prime} \in B_{R}\left(p ;\left(X, d_{X, \chi}\right)\right)$ with $d_{X, \chi}\left(y, y^{\prime}\right)<4 \varepsilon_{5}$. We may also easily verify that for any $y \in B_{R}\left(p ;\left(X, d_{X, \chi}\right)\right)$ there exists $x \in B_{R}(p ; M)$ with

$$
d_{X, \chi}(y, \Psi(x))<2 \varepsilon_{5} .
$$

Then modifying $\Psi$ slightly we see that $d_{G H}\left(B_{R}(p ; M), B_{R}\left(p ;\left(X, d_{X, \chi}\right)\right)<9 \varepsilon_{5}\right.$. Note that $\varepsilon_{5}$ may be arbitrarily small if we take $\varepsilon=\varepsilon(\chi, R)$ sufficiently small. This completes the proof of Theorem 1.1.

Note that in the proof above we only need that the Ricci curvature conditions $\operatorname{Ric}_{M} \geq-(m-1) \kappa, \operatorname{Ric}_{M}(\nabla f, \nabla f) \geq-(m-1) \delta$ and $(2.8)$ hold on sufficiently large concentric distance ball $B_{\tilde{R}}(p ; M)$ of radius $\tilde{R}$, e.g. $\tilde{R}=16 R$, compared with $B_{R}(p ; M)$.

Finally, we give an application. Let $\left\{\left(\left(M_{i}, p_{i}\right), f_{i}\right)\right\}$ be a sequence of pairs of pointed complete connected Riemannian $m$-manifolds $\left(M_{l}, p_{i}\right)$ and smooth functions $f_{i}$ on $M_{l}$ with $\left\|\nabla f_{i}\right\| \equiv 1$ such that $p_{i} \in f_{l}^{-1}(0)$ and their Ricci curvatures satisfy $\operatorname{Ric}_{M_{i}} \geq-(m-1) \kappa, \operatorname{Ric}_{M_{i}}\left(\nabla f_{i}, \nabla f_{i}\right) \geq-(m-1) \delta_{i}$. Suppose $\delta_{i} \downarrow 0$. Then for fixed $R>0,\left\{B_{R}\left(p_{i} ; M_{l}\right)\right\}$ are uniformly compact in the sense of Gromov ([G]) and so is $\left\{B_{R}\left(p_{i} ;\left(Z_{l}, d_{\chi_{i}}\right)\right)\right\}$ with above chosen $\chi_{l} \rightarrow 0$. From Gromov precompactness theorem we may assume that $\left\{\left(Z_{l}, p_{i}\right)\right\}$ converges to a pointed metric space $(Z, p)$ in the pointed Gromov-Hausdorff topology taking a subsequence if necessary. It follows that $\left\{\left(M_{l}, p_{i}\right)\right\}$ converge to $(X=\boldsymbol{R} \times Z, p)$ by Theorem 1.1. Note that we may choose Hausdorff approximations $\Psi_{l}: M_{l} \rightarrow$ $X_{l}=\boldsymbol{R} \times Z_{l}$ so that $f_{i}=\underline{r}_{l} \circ \Psi_{l}$, where $\underline{r}_{l}: X_{l} \rightarrow \boldsymbol{R}$ denotes the canonical projections. This roughly means that $f_{i}$ converge to $\underline{r}: X \rightarrow \boldsymbol{R}$, the canonical projection to the first factor $\boldsymbol{R}$ of the product $X$. Therefore we have the following:

Remark 2.6. In the situation above any pointed Gromov-Hausdorff limit $X$ of $\left\{\left(M_{l}, p_{i}\right)\right\}$ splits $X=\boldsymbol{R} \times Z$ as a direct product of metric spaces. Furthermore, $f_{i}$ converge to the function $\underline{r}: X \rightarrow \boldsymbol{R}$ in the sense above. 


\section{Proof of Theorem 1.2}

First we remark the following elementary fact.

LEMMA 3.1. Let $(M, g)$ be a complete connected Riemannian manifold admitting a function $f$ with $\|\nabla f\| \equiv 1$. Let $Z: f^{-1}(0)$ be endowed with the induced Riemannian metric. Then $M$ is isometric to a warped product metric $R \times_{\psi} Z$ with $\psi(0)=1$ so that $f$ corresponds to the canonical projection onto the first factor if and only if there exists a function $k: \boldsymbol{R} \rightarrow \boldsymbol{R}$ such that

$$
D^{2} f=(k \circ f)\{g-d f \otimes d f\},
$$

where $k, \psi$ are related by $k(s)=\psi^{\prime}(s) / \psi(s)$ or $\psi(s)=\exp \left(\int_{0}^{t} k(t) d t\right)$.

Proof. Suppose $M$ is isometric to $R \times_{\psi} Z$ in the above way. Then for any $u \in U_{p} Z, p \in Z$ we get

$$
\left\langle D \varphi_{s} u, D \varphi_{s} u\right\rangle=\psi^{2}(s)\langle u, u\rangle \text {. }
$$

Differentiating this equation with respect to $s$, we obtain

$$
D^{2} f\left(D \varphi_{s} u, D \varphi_{s} u\right)=\frac{\psi^{\prime}(s)}{\psi(s)}\left\langle D \varphi_{s} u, D \varphi_{s} u\right\rangle .
$$

Since $\nabla f$ is orthogonal to $D \varphi_{s} u$ and belongs to the null space of the Hessian $D^{2} f$, it follows that

$$
D^{2} f_{\varphi_{s}(p)}=\frac{\psi^{\prime}(s)}{\psi(s)}\{g-d f \otimes d f\} .
$$

Conversely, suppose $D^{2} f=(k \circ f)\{g-d f \otimes d f\}$. Then we get for $u \in U_{p} Z$, $p \in Z$

$$
\frac{d}{d s} \log \left\langle D \varphi_{s} u, D \varphi_{s} u\right\rangle=2 \frac{D^{2} f\left(D \varphi_{s} u, D \varphi_{s} u\right)}{\left\langle D \varphi_{s} u, D \varphi_{s} u\right\rangle}=2 k(s)
$$

and therefore

$$
\left\langle D \varphi_{s} u, D \varphi_{s} u\right\rangle=\exp \left(2 \int_{0}^{s} k(t) d t\right)=\psi(s)^{2}\langle u, u\rangle
$$

This completes the proof of the Lemma.

For instance, if $k \equiv 0$ we have the Riemannian direct product $M=R \times Z$, and if $|k| \equiv 1$ then $M=R \times{ }_{\psi} Z$ with $\psi(t)=\exp ( \pm t)$. Also note that

$$
k \circ f=-\frac{\Delta f}{m-1} .
$$

Now let $(M, g)$ be a complete connected Riemannian manifold of dimension $m$ with $\operatorname{Ric}_{M}(\nabla f, \nabla f) \geq-(m-1)$, where $f: M \rightarrow \boldsymbol{R}$ is a smooth function with 
$\|\nabla f\| \equiv 1$. Then we recall that $|\Delta f| \leq m-1$, and $|\Delta f| \equiv m-1$ holds if and only if $M$ is isometric to a warped product $X=R \times{ }_{\psi} Z$, where $Z=f^{-1}(0)$ is endowed with the induced Riemannian metric and $\psi(t)=\exp ( \pm t)$. Now in the present section we ask what happens if $\operatorname{Ric}_{M}(\nabla f, \nabla f) \geq-(m-1)-\delta(0<\delta<1)$ and $|\Delta f|$ is close to $m-1$. First we assume a rather strong condition

$$
|\Delta f \pm(m-1)|<\delta, \quad \nabla f(\Delta f)<\delta .
$$

Then by the Bochner formula we get

$$
\begin{gathered}
\left\|D^{2} f+\frac{\Delta f}{m-1}(g-d f \otimes d f)\right\|^{2}=\left\|D^{2} f\right\|^{2}-\frac{(\Delta f)^{2}}{m-1} \\
=-\operatorname{Ric}_{M}(\nabla f, \nabla f)+\langle\nabla f, \nabla \Delta f\rangle-\frac{(\Delta f)^{2}}{m-1} \\
<2 \delta+(m-1)\left\{1-\frac{(\Delta f)^{2}}{(m-1)^{2}}\right\}<5 \delta .
\end{gathered}
$$

For $u \in U_{p} M, p \in Z$, it follows that

$$
\begin{aligned}
& \left|\frac{d}{d s} \log \left\langle D \varphi_{s} u, D \varphi_{s} u\right\rangle \mp 2\right|=2\left|\frac{D^{2} f\left(D \varphi_{s} u, D \varphi_{s} u\right)}{\left\langle D \varphi_{s} u, D \varphi_{s} u\right\rangle} \mp 1\right| \\
& \quad \leq 2\left|\left\{D^{2} f+\frac{\Delta f}{m-1}(g-d f \otimes d f)\right\}\left(\frac{D \varphi_{s} u}{\left\|D \varphi_{s} u\right\|}, \frac{D \varphi_{s} u}{\left\|D \varphi_{s} u\right\|}\right)\right|+2\left|\frac{\Delta f}{m-1} \pm 1\right| \\
& \quad<7 \sqrt{\delta},
\end{aligned}
$$

and therefore integrating the inequality above we obtain

$$
\exp ( \pm s) \cdot \exp (-4 \sqrt{\delta} s) \leq\left\|D \varphi_{s} u\right\| \leq \exp ( \pm s) \cdot \exp (4 \sqrt{\delta} s)
$$

namely, for the operator norm of $D \Phi: T_{(s, z)} X \rightarrow T_{\Phi(s, z)} M$

$$
\exp (-4 \sqrt{\delta} s) \leq\left\|D \Phi_{(s, z)}\right\| \leq \exp (4 \sqrt{\delta} s),
$$

where $X=\boldsymbol{R} \times Z$ is endowed with the warped product metric with warping function $\psi(t)=\exp ( \pm t)$, and $Z$ is endowed with the induced metric. Thus we proved

Proposition 3.2. Suppose $M$ and $f$ with $\|\nabla f\| \equiv 1$ satisfy $\operatorname{Ric}_{M}(\nabla f, \nabla f) \geq$ $-(m-1)-\delta$ and (3.3). Then for any $\varepsilon>0$ and any $R>0$ there exists $\tau=\tau(\varepsilon, m, R)>0$ such that if $0<\delta<\tau$, then for $\Phi$ restricted to $B_{R}(p ; X) \subset X$, $p \in Z$ we have

$$
(1-\varepsilon) d_{X}(x, y) \leq d_{M}(\Phi(x), \Phi(y)) \leq(1+\varepsilon) d_{X}(x, y),
$$

where $X=\boldsymbol{R} \times_{\psi} Z$ is a warped product metric with $\psi(t)=\exp ( \pm t)$ and the induced metric on $Z$. Furthermore, levels of $f$ are almost totally umbilical in the sense of (3.4). 
In fact, to get (3.5) it suffices to assume that $\operatorname{Ric}_{M}(\nabla f, \nabla f) \geq-(m-1)-\delta$ and (3.3) hold on a larger concentric distance ball $B_{2 R}(p ; M)$.

Again, the assumption (3.3) is geometrically not nice, and now we consider complete connected Riemannian $m$-manifold $M$ admitting a function $f$ with $\|\nabla f\| \equiv 1$ which satisfies

$$
\operatorname{Ric}_{M} \geq-(m-1) \kappa, \quad \operatorname{Ric}_{M}(\nabla f, \nabla f) \geq-(m-1)-\delta
$$

on $B_{2 R}(p ; M)$ and

$$
\frac{1}{\operatorname{vol} B_{2 R}(p ; M)} \int_{B_{2 R}(p ; M)}|\Delta f \pm(m-1)|^{2} d v_{g}<\delta^{2} .
$$

In the following we take plus sign $\Delta f+(m-1)$ in the integrand of (3.7) which corresponds to the case where the model space is the warped product space $\boldsymbol{R} \times{ }_{\psi} Z$ with warping function $\psi(t)=\exp t . \Delta f-(m-1)$ in (3.7) corresponds to the case with $\psi(t)=\exp (-t)$. Now we show the following lemma.

Lemma 3.3. Suppose that (3.6), (3.7) hold on $B_{2 R}(p ; M), p \in M$. Then we have

$$
\frac{1}{\operatorname{vol} B_{R}(p ; M)} \int_{B_{R}(p ; M)}\left\|D^{2} f-(g-d f \otimes d f)\right\|^{2} d v_{g}<C \delta,
$$

where $C=C(m, \kappa, R)$ is a positive constant.

Proof. Take a function $\tilde{f}$ such that $\Delta \tilde{f} \equiv 1-m$ on $B_{2 R}(p ; M)$. Now from the Bochner formula we obtain

$$
\left\|D^{2} f\right\|^{2}=-\operatorname{Ric}_{M}(\nabla f, \nabla f)+\langle\nabla \Delta f, \nabla f\rangle \leq m-1+\delta+\langle\nabla \Delta f, \nabla f\rangle .
$$

Then taking a cut off function $\phi$ as in (2.10) we get

$$
\begin{aligned}
\phi\langle\nabla \Delta f, \nabla f\rangle & =\phi\langle\nabla(\Delta f-\Delta \tilde{f}), \nabla f\rangle \\
& =\operatorname{div}(\phi \Delta(f-\tilde{f}) \nabla f)-\Delta(f-\tilde{f})\langle\nabla \phi, \nabla f\rangle+\phi \Delta f \Delta(f-\tilde{f}) .
\end{aligned}
$$

Now note that we have

$$
\begin{aligned}
& \int_{B_{2 R}(p ; M)}|\Delta f-\Delta \tilde{f}| d v_{g} \leq \delta \operatorname{vol} B_{2 R}(p ; M) \\
& \int_{B_{2 R}(p ; M)}|\Delta f|^{2} d v_{g} \leq 2\left\{\delta^{2}+(m-1)^{2}\right\} \operatorname{vol} B_{2 R}(p ; M)
\end{aligned}
$$

by (3.7). It follows from the Green theorem, Cauchy-Schwarz inequality and Bishop-Gromov volume comparison theorem that 


$$
\begin{gathered}
\int_{B_{2 R}(p ; M)} \phi\left\{\left\|D^{2} f\right\|^{2}-(m-1)\right\} d v_{g} \leq\left|\int_{B_{2 R}(p ; M)} \phi\{\langle\nabla \Delta f, \nabla f\rangle+\delta\} d v_{g}\right| \\
\leq \delta \operatorname{vol} B_{2 R}(p ; M)+c_{1}(m, \kappa, R) \sqrt{\operatorname{vol} B_{2 R}(p ; M) \int_{B_{2 R}(p)}|\Delta f-\Delta \tilde{f}|^{2} d v_{g}} \\
\leq \delta c_{2}(m, \kappa, R) \operatorname{vol} B_{2 R}(p ; M) \leq \delta c(m, \kappa, R) \operatorname{vol} B_{R}(p ; M)
\end{gathered}
$$

as in Lemma 2.2, and hence

$$
\begin{aligned}
& \frac{1}{\operatorname{vol} B_{R}(p ; M)} \int_{B_{R}(p ; M)}\left\|D^{2} f-(g-d f \otimes d f)\right\|^{2} d v_{g} \\
& \quad \leq \frac{1}{\operatorname{vol} B_{R}(p ; M)} \int_{B_{2 R}(p ; M)} \phi\left\|D^{2} f-(g-d f \otimes d f)\right\|^{2} d v_{g} \\
& \quad=\frac{1}{\operatorname{vol} B_{R}(p ; M)} \int_{B_{2 R}(p ; M)} \phi\left\{\left\|D^{2} f\right\|^{2}-(m-1)+2(\Delta f-\Delta \tilde{f})\right\} d v_{g} \\
& \quad<C(m, \kappa, R) \delta .
\end{aligned}
$$

This completes the proof of the Lemma.

Now let $\gamma=\gamma_{y_{1} y_{2}}$ be a minimal geodesic joining $y_{1}$ to $y_{2}$ in $M$ parametrized by arclength. As in (2.13) we set $\mathscr{U}(s)=\mathscr{U}\left(s ; y_{1}, y_{2}\right):=f(\gamma(s))$, and get (2.14). We want to compare $\mathscr{U}(s)$ with the corresponding $\mathscr{U}(s)$ satisfying the same boundary condition in the model space $X=\boldsymbol{R} \times{ }_{\psi} Z$, where $\psi(t)=\exp t$ and $Z=f^{-1}(0)$ is endowed with a Riemannian metric. Note that for $\underline{f}(x):=\underline{r}(x)$, the projection onto the first factor, we obtain

$$
\Delta \underline{f}=1-m, \quad D^{2} \underline{f}=\underline{g}-\underline{d f} \otimes \underline{d f},
$$

where $g$ denotes the warped product metric on $X$ with warping function $\psi$. Again as in the previous section, for some function $\rho: \boldsymbol{R}^{+} \times \boldsymbol{R}^{+} \times \boldsymbol{R}^{+} \rightarrow \boldsymbol{R}^{+}$we may write

$$
d_{X}\left(\left(r_{1}, z_{1}\right),\left(r_{2}, z_{2}\right)\right)=\rho\left(r_{1}, r_{2}, d_{Z}\left(z_{1}, z_{2}\right)\right) .
$$

Next suppose that $\underline{x}_{l}, \underline{y}_{l} \in X(i=1,2)$ satisfy $\underline{\pi}\left(\underline{x}_{1}\right)=\underline{\pi}\left(\underline{y}_{1}\right), \underline{\pi}\left(\underline{x}_{2}\right)=\underline{\pi}\left(\underline{y}_{2}\right)$. Then we may write the distance function $d_{X}$ in the form

$$
d_{X}\left(\underline{y}_{1}, \underline{y}_{2}\right)=Q\left(\underline{r}\left(\underline{x}_{1}\right), \underline{r}\left(\underline{y}_{1}\right), \underline{r}_{(}\left(\underline{x}_{2}\right), \underline{r}_{2}\left(\underline{y}_{2}\right), d_{X}\left(\underline{x}_{1}, \underline{x}_{2}\right)\right) .
$$

See e.g., [C-C-1] for general warped product case. In our special case, we have

$$
\cosh \rho\left(r_{1}, r_{2}, v\right)=\frac{\exp \left(r_{1}+r_{2}\right)}{2} v^{2}+\cosh \left(r_{2}-r_{1}\right)
$$

and 


$$
\begin{aligned}
\cosh d_{X}\left(\underline{y}_{1}, \underline{y}_{2}\right)= & \cosh d_{X}\left(\underline{x}_{1}, \underline{x}_{2}\right) \exp \left(\underline{r}\left(\underline{y}_{1}\right)+\underline{r}\left(\underline{y}_{2}\right)-\underline{r}\left(\underline{x}_{1}\right)-\underline{r}\left(\underline{x}_{2}\right)\right) \\
& -\sinh \left(\underline{r}_{y}\left(\underline{y}_{1}\right)-\underline{r}\left(\underline{x}_{1}\right)\right) \exp \left(\underline{r}\left(\underline{y}_{2}\right)-\underline{r}\left(\underline{x}_{1}\right)\right) \\
& -\sinh \left(\underline{r}\left(\underline{y}_{2}\right)-\underline{r}\left(\underline{x}_{2}\right)\right) \exp \left(\underline{r}\left(\underline{y}_{1}\right)-\underline{r}\left(\underline{x}_{2}\right)\right) .
\end{aligned}
$$

Then it easily follows that for $z_{1}, z_{2} \in Z$

$$
\left\{\begin{array}{l}
d_{Z}\left(z_{1}, z_{2}\right)=2 \sinh \frac{d_{X}\left(z_{1}, z_{2}\right)}{2} \\
\rho\left(r_{1}, r_{2}, 2 \sinh \frac{d_{X}\left(z_{1}, z_{2}\right)}{2}\right)=Q\left(0, r_{1}, 0, r_{2}, d_{X}\left(z_{1}, z_{2}\right)\right) .
\end{array}\right.
$$

We briefly explain how to get (3.12), (3.13).

Let $\underline{\gamma}:[0, l] \rightarrow X$ be a geodesic in $X$. We set $\underline{x}(s):=\underline{\mathscr{U}}^{\prime}(s)$, where $\underline{\mathscr{U}}(s):=\underline{f}(\underline{\gamma}(s))$. Then we have

$$
\underline{\dot{x}}(s)=D^{2} \underline{f}(\underline{\dot{\gamma}}(s), \underline{\dot{\gamma}}(s))=1-\underline{x}^{2}(s) .
$$

Solving this equation under the boundary condition $r_{0}=\underline{\mathscr{U}}(0), \quad r_{l}=\underline{\mathscr{U}}(l)$ $\left(\left|r_{l}-r_{0}\right| \leq l\right)$, we get

$$
\begin{gathered}
\underline{\mathscr{U}}(s)\left(=\underline{\mathscr{U}}\left(s ; r_{0}, r_{l}, l\right)\right)=\log \frac{\exp r_{0} \sinh (l-s)+\exp r_{l} \sinh s}{\sinh l} \\
=: \underline{f}\left(s ; r_{0}, r_{l}, l\right), \\
\underline{\mathscr{U}^{\prime}}(s)=\frac{\exp r_{l} \cosh s-\exp r_{0} \cosh (l-s)}{\exp r_{l} \sinh s+\exp r_{0} \sinh (l-s)} \\
=: \cos \underline{\theta}(s), \quad \underline{\theta}(s)=: \Theta\left(s ; r_{0}, r_{l}, l\right) .
\end{gathered}
$$

Then (3.13) may be obtained by solving the corresponding differential equations for the warped product case using (3.15), (3.16) (see (3.21) below). Note that this formula is nothing but the one for the hyperbolic plane $H^{2}=\boldsymbol{R} \times{ }_{\psi} \boldsymbol{R}$ with $\psi(t)=\exp t$, since these differential equations do not depend on particular $Z$. To show (3.12) it suffices to consider in $H^{2}=\boldsymbol{R} \times{ }_{\psi} \boldsymbol{R}$. We write a geodesic $\gamma(s), 0 \leq s \leq l$, in $H^{2}$ parametrized by arclength joining $\left(r_{1}, z_{1}\right)$ to $\left(r_{2}, z_{2}\right)$ in the form $\underline{\gamma}(s)=(\underline{f}(s), \underline{y}(s)) \in \boldsymbol{R} \times{ }_{\psi} \boldsymbol{R}$. Observe that

$$
\|\underline{\dot{y}}(s)\|_{R}=\exp (-\underline{\mathcal{U}}(s)) \sqrt{1-\underline{\mathcal{U}}^{\prime}(s)^{2}} .
$$

Then we get

$$
\begin{aligned}
d_{Z}\left(z_{1}, z_{2}\right)= & \sinh l \sqrt{2 \exp \left(r_{1}+r_{2}\right) \cosh l-\left(\exp \left(2 r_{1}\right)+\exp \left(2 r_{2}\right)\right)} \\
& \times \int_{0}^{l} \frac{d s}{\left(\exp r_{1} \sinh (l-s)+\exp r_{2} \sinh s\right)^{2}} \\
= & \frac{1}{\exp \left(r_{1}+r_{2}\right)} \sqrt{2 \exp \left(r_{1}+r_{2}\right) \cosh l-\left(\exp \left(2 r_{1}\right)+\exp \left(2 r_{2}\right)\right)},
\end{aligned}
$$

from which (3.12) follows. 
Then the formulas (3.12), (3.13), $(3,14)$ determine the warped product distance on $X$ with $\psi(t)=\exp t$, once a distance is given on $Z$. Also note that for fixed $r_{0}, r_{N},\left\{v_{i}\right\}_{l=1}^{N}$ there exist $r_{1}, \ldots, r_{N-1}$ such that

$$
\sum_{l=0}^{N-1} \rho\left(r_{l}, r_{l+1}, v_{i+1}\right)=\rho\left(r_{0}, r_{N}, \sum_{l=1}^{N} v_{i}\right) .
$$

Now we compare $\mathscr{U}(s)=\mathscr{U}\left(s ; y_{1}, y_{2}\right)$ with $\underline{\mathscr{U}}(s)=\underline{\mathscr{U}}\left(s ; r_{0}, r_{l}, l\right)$ setting $r_{0}=f\left(y_{1}\right)=\mathscr{U}(0), r_{l}=f\left(y_{2}\right)=\mathscr{U}(l), l=d\left(y_{1}, y_{2}\right)$. First we assume that

$$
\int_{0}^{l}\left|\mathscr{U}^{\prime \prime}(s)+\left(\mathscr{U}^{\prime}(s)\right)^{2}-1\right| d s<\varepsilon_{2} .
$$

Setting $\beta(s):=\mathscr{U}^{\prime \prime}(s)+\left(\mathscr{U}^{\prime}(s)\right)^{2}-1$ and $y(s):=\exp \mathscr{U}(s), \underline{y}(s):=\exp \underline{\mathscr{U}}(s)$, we have

$$
y^{\prime \prime}(s)=(\beta(s)+1) y(s), \quad \underline{y}^{\prime \prime}(s)=\underline{y}(s), \quad y(0)=\underline{y}(0), \quad y(l)=\underline{y}(l) .
$$

Note that since $\left|\mathscr{U}^{\prime}(s)\right|,\left|\underline{\mathcal{U}}^{\prime}(s)\right| \leq 1$ we have uniform bounds $|\mathscr{U}(s)|,|\underline{\mathscr{U}}(s)| \leq$ $C\left(l, r_{0}\right), 0 \leq s \leq l$. Now we set $z=y-y$ and get $z^{\prime \prime}(s)=z(s)+\alpha(s)$ with $\alpha(s)=\beta(s) y(s)$. Note that $z(0)=z(l)=0$. It follows that

$$
\begin{aligned}
\int_{0}^{l}\left\{z^{\prime}(s)^{2}+z(s)^{2}\right\} d s & =z^{\prime}(l) z(l)-z^{\prime}(0) z(0)-\int_{0}^{l} z(s) \alpha(s) d s \\
& \leq \psi\left(\varepsilon_{2} \mid r_{0}, l\right)\left(\leq \psi\left(\varepsilon_{2} \mid R_{1}\right)\right) .
\end{aligned}
$$

From the above we easily see that $|z(s)|$ is small enough and $\left|z^{\prime}(s)-z^{\prime}(0)\right|<$ $\psi\left(\varepsilon_{2} \mid R_{1}\right)$. It follows that $\left|z^{\prime}(s)\right|$ is also small, namely we get

$$
\left|\mathscr{U}^{\prime}(s)-\underline{\mathscr{U}}^{\prime}(s)\right|, \quad|\mathscr{U}(s)-\underline{\mathscr{U}}(s)| \leq \psi\left(\varepsilon_{2} \mid R_{1}\right) .
$$

Now to compare $\mathscr{U}(s)$ with $\underline{\mathscr{U}}(s)$ for general $\gamma_{y_{1} y_{2}}$ we again appeal to [C-C-1], Theorem 2.11 and get

\section{LEMMA 3.4. Suppose that}

$$
\frac{1}{\operatorname{vol} B_{4 R_{1}}(p)} \int_{B_{4 R_{1}}(p)}\left\|D^{2} f-(g-d f \otimes d f)\right\| d v_{g} \leq \varepsilon_{1} .
$$

Then there exists $C\left(m, \kappa, R_{1}\right)>0$ such that

$$
\begin{aligned}
& \frac{1}{\left(\operatorname{vol} B_{2 R_{1}}(p)\right)^{2}} \int_{B_{2 R_{1}}(p) \times B_{2 R_{1}}(p)} d v_{g \oplus g} \int_{0}^{l}\left|\mathcal{U}^{\prime \prime}(s)+\mathscr{U}^{\prime}(s)^{2}-1\right| d s \\
& \quad \leq C\left(m, \kappa, R_{1}\right) \varepsilon_{1}
\end{aligned}
$$

holds, where we set $\mathscr{U}(s)=\mathscr{U}\left(s ; y_{1}, y_{2}\right)$ and $l=d_{M}\left(y_{1}, y_{2}\right)$.

Again note that from Lemma 3.3, (3.19) holds for any $4 R_{1}>0$ taking $\delta=\delta\left(m, \kappa, R_{1}\right)$ in (3.8) sufficiently small. 
Then we may argue as before with corresponding changes in Lemma 2.5 to get the following Lemma 3.5. For instance, $\underline{l}(s)$ in this case is given by

$$
\cosh \underline{l}(s)=\cosh \underline{l}(0) \exp s-\exp \left(f(x)-f\left(x_{2}\right)\right) \sinh s
$$

instead of $\underline{l}(s)=\sqrt{(s+a)^{2}+b^{2}}$. Indeed, (3.21) may be obtained by solving the differential equation

$$
\begin{aligned}
\underline{l}^{\prime}(s) \sinh \underline{l}(s) & =\cos \Theta\left(\underline{l}(s) ; f(x), f\left(x_{2}\right)+s, \underline{l}(s)\right) \sinh \underline{l}(s) \\
& =\cosh \underline{l}(s)-\exp \left(f(x)-f\left(x_{2}\right)\right) \exp (-s) .
\end{aligned}
$$

We consider

$$
\int_{0}^{d} \sinh l(s)\left|\mathscr{U}^{\prime}(l(s) ; y, \lambda(s))-\underline{\mathscr{U}}^{\prime}(s)(l(s) ; f(y), f(\lambda(s)), l(s))\right| d s<\eta^{2}
$$

instead of (2.25). Note also that

$$
\begin{aligned}
\underline{\mathscr{U}}(s, f(q), f(w), d) & =\log \frac{\exp f(q) \cdot \sinh (d-s)+\exp f(w) \cdot \sinh s}{\sinh d}, \\
\underline{\mathscr{U}}^{\prime}(l(s) ; f(y), f(\lambda(s)), l(s)) & =\cos \Theta(l(s) ; f(y), f(\lambda(s)), l(s)) \\
& =\frac{\exp f(\lambda(s)) \cdot \cosh l(s)-\exp f(y)}{\exp f(\lambda(s)) \cdot \sinh l(s)}
\end{aligned}
$$

and that

$$
l^{\prime}(s)=\cos \alpha(s), \quad \alpha(s):=\angle\left(\dot{\gamma}_{y \lambda(s)}(l(s)), \dot{\lambda}(s)\right)
$$

holds almost everywhere with

$$
\left|\cos \alpha(s)-\mathscr{U}^{\prime}(l(s) ; y, \lambda(s))\right|<\psi\left(\varepsilon_{2}, \eta \mid R_{1}\right) .
$$

Then setting $w(s):=\cosh l(s)-\cosh \underline{l}(s)$ and recalling that

$$
\cos \Theta(l(s) ; f(y), f(\lambda(s)), l(s)) \sinh l(s)=\cosh l(s)-\exp (f(y)-f(\lambda(s))) \text { etc. },
$$

we obtain

$$
\begin{aligned}
w^{\prime}(s)-w(s)= & \sinh l(s)(\cos \alpha(s)-\cos \Theta(l(s) ; f(y), f(\lambda(s)), l(s))) \\
& -\sinh \underline{l}(s)(\cos \underline{\alpha}(s)-\cos \Theta(\underline{l}(s) ; \underline{f}(\underline{y}) \underline{f}(\underline{\lambda}(s)), \underline{l}(s))) \\
& -\exp (f(y)-f(\lambda(s)))+\exp \underline{f}(\underline{y})-\underline{f}(\underline{\lambda}(s)))
\end{aligned}
$$

almost everywhere. It follows that

$$
\int_{0}^{d}\left|w^{\prime}(s)-w(s)\right| d s<\psi\left(\varepsilon_{2}, \eta \mid R_{1}\right)
$$

with $\quad w(0)=0$ as before. Considering $\exp (-s) w(s)$ we obtain $|w(d)|<$ $\psi\left(\varepsilon_{2}, \eta \mid R_{1}\right)$ by integration, and therefore $|l(d)-\underline{l}(d)|<\psi\left(\varepsilon_{2}, \eta \mid R_{1}\right)$. 
LEMMA 3.5. For any $\varepsilon>0$ there exists $\zeta=\zeta\left(\varepsilon, m, \kappa, R_{1}\right)>0$ such that if

$$
\frac{1}{\operatorname{vol} B_{4 R_{1}}(p)} \int_{B_{4 R_{1}}(p)}\left\|D^{2} f-(g-d f \otimes d f)\right\| d v_{g}<\varepsilon_{1}
$$

holds for $0<\varepsilon_{1}<\zeta$, then we have

$$
\left|d_{M}\left(y_{1}, y_{2}\right)-Q\left(f\left(x_{1}\right), f\left(y_{1}\right), f\left(x_{2}\right), f\left(y_{2}\right), d_{M}\left(x_{1}, x_{2}\right)\right)\right|<\varepsilon
$$

for any $x_{i}, y_{i} \in B_{R_{1}}(p)$ with $\left|f\left(y_{i}\right)-f\left(x_{l}\right)\right|=d\left(x_{i}, y_{i}\right)(i=1,2)$.

Now for small $(1>) \chi>0$ we define a distance $d_{\chi}$ on $Z$ by (2.30). Then on $X$ we define a distance $d_{X, \chi}$ by

$$
d_{X, \chi}\left((r, z),\left(r^{\prime}, z^{\prime}\right)\right):=\rho\left(r, r^{\prime}, d_{\chi}\left(z, z^{\prime}\right)\right)
$$

which is in fact a warped product distance with warping function $\psi(t)=\exp t$. Here note that

$$
\begin{aligned}
\rho\left(r, r^{\prime}, d_{M}\left(z, z^{\prime}\right)\right) & \leq Q\left(0, r, 0, r^{\prime}, d_{M}\left(z, z^{\prime}\right)\right) \\
& \leq \rho\left(r, r^{\prime}, d_{M}\left(z, z^{\prime}\right)\right)+\frac{d_{M}\left(z, z^{\prime}\right)^{3}}{9} \exp \left(\frac{r+r^{\prime}}{2}\right)
\end{aligned}
$$

holds if $d_{M}\left(z, z^{\prime}\right)<1$ by (3.14).

Now our aim is to show that

$$
\left|d_{M}\left(x, x^{\prime}\right)-d_{X, \chi}\left(\Psi(x), \Psi\left(x^{\prime}\right)\right)\right|<\psi(\varepsilon|\chi| R)
$$

for $x, x^{\prime} \in B_{R}(p ; M)$, where $\varepsilon>0$ in (3.22) may become arbitrary small if we choose $\delta$ in (3.8) sufficiently small. For that purpose we take a minimal geodesic $\gamma=\gamma_{x x^{\prime}}$ of $M$ parametrized by arclength and a subdivision $\left\{\gamma\left(s_{i}\right) ; s_{i}=i l / N\right.$, $i=0, \ldots, N\}$ of $\gamma$, where we set $l=d\left(x, x^{\prime}\right)$. Note that $\gamma\left(s_{i}\right) \in B_{2 R}(p ; M)$, $\pi\left(\gamma\left(s_{i}\right)\right) \in B_{4 R}(p ; M)$. Then applying Lemma 3.5 with $R_{1}=4 R$ we obtain

$$
\left|d_{M}\left(\pi\left(\gamma\left(s_{i}\right)\right), \pi\left(\gamma\left(s_{i+1}\right)\right)\right)-Q\left(f\left(\gamma\left(s_{i}\right)\right), 0, f\left(\gamma\left(s_{i+1}\right)\right), 0, d_{M}\left(\gamma\left(s_{i}\right), \gamma\left(s_{i+1}\right)\right)\right)\right|<\varepsilon .
$$

Taking a sufficiently large $N:=N(\chi, R)$ it follows that

$$
d_{M}\left(\pi\left(\gamma\left(s_{i}\right)\right), \pi\left(\gamma\left(s_{i+1}\right)\right)\right)<\chi .
$$

Note that $d_{\chi}\left(\pi\left(\gamma\left(s_{i}\right)\right), \pi\left(\gamma\left(s_{i+1}\right)\right)\right)=d_{M}\left(\pi\left(\gamma\left(s_{i}\right)\right), \pi\left(\gamma\left(s_{i+1}\right)\right)\right)$. Then from Lemma 3.5 and (3.26) we obtain

$$
\begin{aligned}
d_{M}\left(x, x^{\prime}\right) & =\sum_{i=0}^{N-1} d_{M}\left(\gamma\left(s_{i}\right), \gamma\left(s_{i+1}\right)\right) \\
& \geq \sum_{i=0}^{N-1} Q\left(0, f\left(\gamma\left(s_{i}\right)\right), 0, f\left(\gamma\left(s_{i+1}\right)\right), d_{M}\left(\pi\left(\gamma\left(s_{i}\right)\right), \pi\left(\gamma\left(s_{i+1}\right)\right)\right)\right)-\varepsilon N \\
& \geq \sum_{i=0}^{N-1} \rho\left(f\left(\gamma\left(s_{i}\right)\right), f\left(\gamma\left(s_{i+1}\right)\right), d_{\chi}\left(\pi\left(\gamma\left(s_{i}\right)\right), \pi\left(\gamma\left(s_{i+1}\right)\right)\right)\right)-\varepsilon N
\end{aligned}
$$




$$
\begin{aligned}
& =\sum_{i=0}^{N-1} d_{X, \chi}\left(\left(f\left(\gamma\left(s_{i}\right)\right), \pi\left(\gamma\left(s_{i}\right)\right)\right),\left(f\left(\gamma\left(s_{i+1}\right)\right), \pi\left(\gamma\left(s_{i+1}\right)\right)\right)\right)-\varepsilon N \\
& \geq d_{X, \chi}\left(\Psi(x), \Psi\left(x^{\prime}\right)\right)-\varepsilon N
\end{aligned}
$$

where $\varepsilon N$ may be arbitrarily small if we take $\varepsilon>0$ sufficiently small. On the other hand, for $x, x^{\prime} \in B_{R}(p ; M)$ set $(r, z):=\Psi(x),\left(r^{\prime}, z^{\prime}\right):=\Psi\left(x^{\prime}\right)$. Then from the definition of the distance $d_{\chi}$ there exist points $z_{i}\left(i=0, \ldots, N_{1}\right) \in Z$ with $z_{0}=z, z_{N_{1}}=z^{\prime}$ such that

$$
0 \leq \sum_{l=0}^{N_{1}-1} d_{M}\left(z_{l}, z_{l+1}\right)-d_{\chi}\left(z, z^{\prime}\right)<\varepsilon, \quad d_{M}\left(z_{l}, z_{l+1}\right)<\chi .
$$

Then there exist points $\left(r_{l}, z_{l}\right) \in \boldsymbol{R} \times Z\left(i=0, \ldots, N_{1}\right)$ with $r_{0}=r, r_{N_{1}}=r^{\prime}$ such that

$$
\sum_{l=0}^{N_{1}-1} \rho\left(r_{l}, r_{l+1}, d_{M}\left(z_{l}, z_{l+1}\right)\right)=\rho\left(r, r^{\prime}, \sum_{l=0}^{N_{1}-1} d_{M}\left(z_{l}, z_{l+1}\right)\right) \text {. }
$$

We set $x_{l}=\Phi\left(r_{l}, z_{l}\right)$, namely, $\left(r_{l}, z_{l}\right)=\Psi\left(x_{l}\right)$. It follows from (3.12) that

$$
\left|d_{X, \chi}\left(\Psi(x), \Psi\left(x^{\prime}\right)\right)-\sum_{l=0}^{N_{1}-1} d_{X, \chi}\left(\Psi\left(x_{l}\right), \Psi\left(x_{l+1}\right)\right)\right|<C \varepsilon,
$$

where $C=C(R)$ is a positive constant. Note that $\left|r_{i}\right|<2 R+C \varepsilon$, $z_{l} \in B_{4 R}(p ; M), x_{l} \in B_{7 R}(p ; M)$. Then Lemma 3.5 with (3.24) implies that

$$
d_{M}\left(x_{l}, x_{l+1}\right)-d_{X, \chi}\left(\left(r_{l}, z_{l}\right),\left(r_{l+1}, z_{l+1}\right)\right)<\varepsilon+C_{1} \chi^{3},
$$

where $C_{1}=C_{1}(R)$ is a positive constant.

Now we give an estimate of $N_{1}$. Noting that $d_{\chi}\left(z_{l}, z_{l+1}\right)+d_{\chi}\left(z_{l+1}, z_{l+2}\right) \geq \chi$ we have

$$
2 d_{\chi}\left(z, z^{\prime}\right) \geq\left(N_{1}-1\right) \chi-2 \varepsilon .
$$

Set $v:=d_{\chi}\left(z, z^{\prime}\right)$. Then from (3.27) we get

$$
\rho\left(r, r^{\prime}, v\right)=d_{X, \chi}\left(\Psi(x), \Psi\left(x^{\prime}\right)\right) \leq 2 R+\chi
$$

taking $\varepsilon>0$ small. Hence noting that $\cosh \rho\left(r, r^{\prime}, v\right) \geq\left(v^{2} / 2\right)+1$ we obtain

$$
v \leq 2 \sinh \frac{\rho\left(r, r^{\prime}, v\right)}{2} \leq 2 \sinh \frac{2 R+\chi}{2} \text {. }
$$

It follows that

$$
N_{1} \leq \frac{4 \sinh (R+\chi)+2 \varepsilon}{\chi}+1
$$

Therefore, we have

$$
\begin{aligned}
d_{M}\left(x, x^{\prime}\right) & \leq \sum_{l=0}^{N_{1}-1} d_{M}\left(x_{l}, x_{l+1}\right) \leq d_{X, \chi}\left(\Psi(x), \Psi\left(x^{\prime}\right)\right)+\left(N_{1}+C\right) \varepsilon+\chi^{2} C_{2}(R) \\
& \leq d_{X, \chi}\left(\Psi(x), \Psi\left(x^{\prime}\right)\right)+\psi(\varepsilon|\chi| R)
\end{aligned}
$$


where $C_{2}=C_{2}(R)$ is a positive constant and for fixed $R>0, \psi(\varepsilon|\chi| R)$ becomes arbitrary small if we take first $\chi>0$ small and then choose $\varepsilon=\varepsilon(\chi)>0$ further small. In the arguments above note that it suffices to assume that (1.8), (1.9) hold on a sufficiently large concentric distance ball $B_{\tilde{R}}(p ; M)$ of radius $\tilde{R}$, e.g. $\tilde{R}=30 R$, compared with the given $B_{R}(p ; M)$. We may proceed with the remaining argument as in the proof of Theorem 1.1, and the proof of Theorem 1.2 is complete.

Remark 3.6. Let $\left\{\left(\left(M_{i}, p_{i}\right), f_{i}\right)\right\}$ be a sequence of pairs of $m$-dimensional complete connected pointed Riemannian manifolds $M_{l}$ and smooth functions $f_{i}$ on $M_{l}$ with $\left\|\nabla f_{i}\right\| \equiv 1$ and $\| \Delta f_{i}|-(m-1)|<\delta_{i}$, where $p_{i} \in Z_{l}:=f_{l}^{-1}(0)$ and their Ricci curvatures satisfy $\operatorname{Ric}_{M_{i}} \geq-(m-1) \kappa, \operatorname{Ric}_{M_{i}}(\nabla f, \nabla f) \geq-(m-1)-\delta_{i}$. Sup$\delta_{i} \downarrow 0$. Then as before we may assume that $\left\{\left(\left(Z_{l}, d_{\chi_{1}}\right), p_{i}\right)\right\}$ with above $\chi_{i}(\downarrow 0)$ converges to a pointed metric space $(Z, p)$ in the pointed Gromov-Hausdorff topology taking a subsequence if necessary. It follows that $\left\{\left(M_{l}, p_{i}\right)\right\}$ converge to $X=R \times{ }_{\psi} Z$ with $\psi(t)=\exp ( \pm t)$ by Theorem 1.2. Therefore, any pointed Gromov-Hausdorff limit $X$ of $\left\{\left(M_{i}, p_{i}\right)\right\}$ splits $X=\boldsymbol{R} \times{ }_{\psi} Z$ as a warped product metric space.

We also note that we may give a similar version of Theorem 1.2 for the case where we take a general warped product space $R \times{ }_{\psi} Z$ with convex $\psi$ as the model ([S-2]).

\section{REFERENCES}

[C-1] T. H. ColdING, Shape of manifolds with positive Riccl curvature, Invent. Math., 144 (1996), 175-191.

[C-2] T. H. Colding, Large manifolds with positive Riccl curvature, Invent. Math., 144 (1996), 193-214.

[C-3] T. H. Colding, Ricci curvature and volume convergence, Ann. of Math., 145 (1997), 477-501.

[C-C-1] J. CheEger AND T. H. Colding, Lower bounds on Ricci curvature and the almost rigidity of warped product, Ann. of Math., 144 (1996), 189-237.

[C-C-2] J. CheEger AND T. H. Colding, On the structure of spaces with Ricci curvature bounded below; I, J. Differential Geom., 46 (1997), 406-480, II, Preprint.

[G] M. GRomov, Groups of polynomial growth and expanding maps, Inst. Hautes Études Sci. Publ. Math., 53 (1981), 53-73.

[S-1] T. SAKAI, On Riemannian manifolds admitting a function whose gradient is of constant norm, Koda1 Math. J., 19 (1996), 39-51.

[S-2] T. SAKAI, Warped products and Riemannian manifolds admitting a function whose gradient is of constant norm, to appear in Math. J. Okayama Univ.

DePartment of MATHEMATICS

FACULTY OF SCIENCE

OKAYAMA UNIVERSITY

OKAYAMA 700-8530, JAPAN

E-mail: saka1@math.okayama-u.ac.jp 